\title{
ENLACES DAS CONVEXIDADES DE CAMINHOS CURTOS EM PROBLEMAS DE PARTIÇÕES E SEPARAÇÕES CONVEXAS
}

\author{
Lorrana F. de Castro \\ Instituto do Noroeste Fluminense / Universidade Federal Fluminense - (INF/UFF) \\ Av. João Jasbick, S/N - Aeroporto - S. A. Pádua/RJ - Brasil \\ lorranafilemes@id.uff.br \\ Rodolfo A. de Oliveira \\ Instituto do Noroeste Fluminense / Universidade Federal Fluminense - (INF/UFF) \\ Av. João Jasbick, S/N - Aeroporto - S. A. Pádua/RJ - Brasil \\ rodolfooliveira@id.uff.br
}

\section{Fábio Protti}

Instituto de Computação / Universidade Federal Fluminense - (IC/UFF)

Gal. Milton Tavares de Souza, S/N - S. Domingos - Niterói/RJ - Brasil

fabio@ic.uff.br

\begin{abstract}
RESUMO
Neste trabalho apresentamos alguns resultados teórico-computacionais sobre a convexidade $P_{3}$ e a convexidade $P_{3}^{*}$ nas perspectivas de partição e separação convexas. Na vertente teórica, mostramos sobre quais aspectos ambas as convexidades são equivalentes, identificamos os menores grafos que não admitem uma partição convexa e listamos infinitos grafos com tal característica e, por último, analisamos os moldes de uma separação para caminhos, árvores, ciclos e cliques. Na vertente computacional, mostramos a NP-completude do problema de partição convexa em ambas as convexidades, mesmo restrito a classes particulares de grafos.
\end{abstract}

Palavra-chave: Convexidade; $P_{3}$-convexidade; Partição Convexa.

\begin{abstract}
In this paper, we present some theoretical and computational results concerning convexity $P_{3}$ and convexity $P_{3}^{*}$ under the convex partitioning and separation perspectives. On the theoretical side, we show which aspects both convexities are equivalent to, we identify the smallest graphs that do not admit a convex partition and list an infinite class of graphs with no such partitioning, and finally, we analyze the patterns of convex separation for paths, trees, cycles, and cliques. In the computational aspect, we show the NP-completeness of the convex partitioning problem in both convexities, even restricted to particular class graphs.
\end{abstract}

Keywords: Convexity; $P_{3}$-convexity; Convex Partitioning.

\section{Como Citar:}

CASTRO, Lorrana F.; OLIVEIRA, Rodolfo A.; PROTTI, Fábio. Enlaces das convexidades de caminhos curtos em problemas de partições e separações convexas em grafos. In: SIMPÓSIO DE PESQUISA OPERACIONAL E LOGÍSTICA DA MARINHA, 19., 2019, Rio de Janeiro, RJ. Anais [...]. Rio de Janeiro: Centro de Análises de Sistemas Navais, 2019. 


\section{INTRODUÇÃO}

O conceito de convexidade, que foi basicamente definido e estudado em $\mathbb{R}^{d}$ nos trabalhos pioneiros de Newton, Minkowski e outros, como descrito em [1], tem encontrado um lugar nas mais diversificadas e distintas estruturas matemáticas. Este desenvolvimento é motivado não apenas pela necessidade de uma teoria abstrata de convexidade generalizando os teoremas clássicos em $\mathbb{R}^{d}$ devidos a Helly, Carathéodory, Radon etc., mas também para unificar aspectos geométricos de todas essas estruturas matemáticas.

Assim, de maneira simples e abrangente, por [2] temos que uma convexidade sobre um conjunto não-vazio $X$ é uma família $\mathscr{C}$ de subconjuntos de $X$ que satisfaz: $\left(c_{1}\right) \emptyset, X \in \mathscr{C} ;\left(c_{2}\right) \mathscr{C}$ é estável por interseções; e $\left(c_{3}\right) \mathscr{C}$ é estável por união aninhada. O par ordenado $(X, \mathscr{C})$ é chamado de espaço de convexidade (estrutura convexa, espaço alinhado), onde os membros de $\mathscr{C}$ são chamados de conjuntos convexos e os seus complementos são chamados de conjuntos côncavos. Note que $\emptyset$ e $X$ são biconvexos triviais. O fecho convexo de $A \subseteq X$, escrito como $[A]_{\mathscr{C}}$, é o menor conjunto convexo contendo $A$. Denote por $2^{X}$ a coleção de todos os subconjuntos de $X$. Um operador intervalar $I: X \times X \rightarrow 2^{X}$ é uma aplicação que segue, para todo $u$ e $v$ em $X$, as seguintes propriedades: $(i) u, v \in I(u, v)$ (Lei da Extensão); (ii) $I(u, v)=I(v, u)$ (Lei da Simetria). O par ordenado $(X, I)$ é chamado de espaço intervalar. Um subconjunto $A$ de $X$ é (intervalo-) convexo se $I(u, v) \subseteq A$, para todo $u, v \in A$. Muitas vezes, o intervalo é uma ferramenta fundamental para criação de espaços de convexidades sobre um dado conjunto.

Muitos problemas e questionamentos ladeiam o tema de convexidade, como por exemplo partição e separação convexa, que são nossos tópicos de interesse. Ainda de [2], um subconjunto $S$ de $X$ é dito biconvexo ou semi-espaço se for convexo e côncavo ao mesmo tempo, i.e., $S$ e $\bar{S}(X \backslash S)$ são ambos convexos. Uma convexidade tem uma separação se satisfaz os seguintes axiomas: $\left(s_{1}\right)$ todos os subconjuntos unitários de $X$ são convexos; $\left(s_{2}\right)$ se $u \neq v \in X$, existe um biconvexo $S$ de $X$ com $u \in S, v \in \bar{S} ;\left(s_{3}\right)$ se $A \subseteq X$ é convexo e $x \in \bar{A}$, então existe um biconvexo $S$ de $X$ com $A \subseteq S$, e $x \in \bar{S}$; $\left(s_{4}\right)$ se $A, B \subseteq X$ são conjuntos convexos disjuntos, então existe um biconvexo $S$ de $X$ com $A \subseteq S$ e $B \subseteq \bar{S}$. Em cada um dos axiomas $s_{2}, s_{3}$ e $s_{4}$, temos um conjunto biconvexo $S$ e dois conjuntos $A$ e $B$, com $A \subseteq S$ e $B \subseteq \bar{S}$. Por tal razão, dizemos que $S$ separa $A$ de $B$. Se $X$ satisfaz o axioma $s_{i}$ então $X$ é chamado de $s_{i}$-espaço de convexidade, e a coleção dos seus conjuntos convexos é chamada de $s_{i}$-convexidade. Podemos generalizar o problema de partição biconvexa no problema de $p$-partição convexas, isto é, verificar a possibilidade de dividir $X$ em $p$ conjuntos disjuntos $\left\{S_{1}, S_{2}, S_{3}, \ldots, S_{p}\right\}$ de modo que $\bigcup S_{i}=X, 1 \leq i \leq p$, e para cada $S_{i}$ vale que $S_{i}$ e $\bar{S}_{i}$ são ambos convexos.

O tema convexidade tem crescido de forma vertiginosa em grafos e seu interesse é atual como podemos observar nos recentes trabalhos [3, 4, 5, 6, 7]. Para um grafo $G=(V, E)$, onde $V$ é o conjunto de vértices e $E$ o conjunto de arestas, em 
grande parte dos trabalhos na área, a convexidade é verificada sobre o conjunto $V$. Com a utilização de métricas para definição de intervalos conforme a característica dos caminhos podemos construir convexidades particulares, e.g., os clássicos intervalos geodésico (para caminhos mínimos) e monofônico (para caminhos induzidos), que constituem, respectivamente, as convexidades Geodésica [8] e Monofônica [9, 10]; intervalo triangulado, que admite caminhos com triângulos, para a convexidade de caminhos triangulados [11]; intervalo de Steiner ${ }^{1}$, que utiliza o conceito de árvore de Steiner, para a convexidade de Steiner [12]. No livro intitulado "Geodesic Convexity in Graphs" [13], podemos encontrar mais informações sobre as convexidades listadas.

A convexidade de nosso interesse é a convexidade que se baseia em caminhos curtos, i.e., caminhos de comprimento exato 2 em grafos. Normalmente, tal convexidade é chamada de convexidade $P_{3}$. Para cada elemento $W \subseteq V$ da convexidade $P_{3}$, temos que qualquer vértice com pelo menos dois vizinhos em $W$ pertence ao mesmo; em outras palavras, não existe nenhum vértice em $V \backslash W$ adjacente a dois vértices em $W$. Uma variação desta é a convexidade $P_{3}^{*}$, a qual exige que o caminho seja induzido, i.e, para todo $W \subseteq V$ na convexidade $P_{3}^{*}$ verifica-se que qualquer vértice com pelo menos dois vizinhos não-adjacentes em $W$ também está em $W$. Ambas as convexidades possuem interesse relevante de pesquisa como podemos observar em alguns trabalhos recentes [14, 15, 16].

Existem diversos interesses computacionais referentes aos parâmetros de uma convexidade [17, 18]. Um desses parâmetros refere-se ao problema de partições convexas, como por exemplo já fora estudado em [19] para convexidade geodésica. O cerne deste trabalho é o estudo do problema de partições convexas não-triviais em grafos na convexidade $P_{3}$ e na sua variação $P_{3}^{*}$. O que motiva a escolha deste tema, além do interesse teórico-computacional, é a sua aplicação hipotética em determinação e delimitação de regiões endêmicas de contágio de alguma enfermidade infectocontagiosa, como por exemplo dengue, leishmaniose ou malária. Se por hipótese assumirmos que uma doença se potencializa a 100\% na sua ação sobre um indivíduo se o mesmo tiver dois tipos de contato por ao menos dois hospedeiros distintos, temos que isso se adéqua à convexidade $P_{3}$. Portanto, dado um grafo que modela indivíduos de uma população e uma região endêmica com uma certa quantidade de indivíduos infectados, podemos tentar encontrar $W$ contendo tais indivíduos da região de modo a ser biconvexo na convexidade $P_{3}$. Assim podemos determinar grupos de indivíduos com potencial de, em algum momento, serem contaminados em $W$, caso não haja controle da doença com os indivíduos na região endêmica; note que em $\bar{W}=V \backslash W$ temos os indivíduos fora de qualquer risco de contágio.

Todavia, verificar se um grafo admite $p$-partição convexa para convexidade $P_{3}$, com $p \geq 2$, é demonstrado ser NP-completo, mesmo para grafos "split" [20]. Neste trabalho, reforçaremos esse resultado mesmo para $p$-fixo, assim como para a convexidade $P_{3}^{*}$. Além disso, mostraremos que o problema é NP-completo para ambas as convexidades restritas a grafos bipartidos e outras classes de grafos. Além

\footnotetext{
${ }^{1}$ Mesmo embora aparente ser degenerado por se basear em árvores, faz sentido quando lembramos que árvore admitem um único caminho entre cada par de vértices.
} 
desses resultados, listaremos infinitos grafos que não admitem uma partição convexa para ambas as convexidades e discutiremos pioneiramente separação convexa em convexidades $P_{3}$ e $P_{3}^{*}$ para classes de grafos pequenas.

\section{DEFINIÇÕES PRELIMINARES}

Considere $G=(V, E)$ um grafo finito, onde $V$ é o conjunto de vértices e $E$ o conjunto de arestas 2 , Denote por $|V|=n$ e $|E|=m$. Um subgrafo $G^{\prime}$ de $G$ deve satisfazer $V\left(G^{\prime}\right) \subseteq V$ e $E\left(G^{\prime}\right) \subseteq E$. Para $W \subseteq V$, o subgrafo induzido por $W$ é um grafo denotado por $G[W]$ de modo que, para todo $u, v \in W$, se existe a aresta $u v \in E$ então $u v \in E(G[W])$. Um grafo $G$ é dito planar quando houver ao menos uma representação de $G$ imersa no plano sem cruzamento de arestas. Dado um vértice $u \in V$, defina a vizinhança aberta de $u$ por $N(u)=\{v \in V: u v \in E\}$, e a vizinhança fechada de $u$ por $N[u]=N(u) \cup\{v\}$. Um vértice $u \in V$ é chamado isolado se $N(u)=\emptyset$. O grau de um vértice $v$ corresponde ao tamanho de $N(v)$. Dois grafos $G_{1}\left(V_{1}, E_{1}\right)$ e $G_{2}\left(V_{2}, E_{2}\right)$ são chamados isomorfos se $\left|V_{1}\right|=\left|V_{2}\right|$ e existe uma função unívoca $f: V_{1} \rightarrow V_{2}$ tal que $u v \in E_{1}$ se, e somente se, $f(u) f(v) \in E_{2}$. Um subconjunto de vértices $W \subseteq V$ é chamado de conjunto estável quando $G[W]$ é constituído apenas por vértices isolados. Usamos os termos partição ou particionar para nos referirmos a qualquer divisão disjunta de elementos de um conjunto, cuja união é exatamente o próprio conjunto. Um grafo admite uma bipartição se podemos particionar seu conjunto de vértices em dois subconjuntos não-vazios e estáveis. Tal grafo é chamado de bipartido. Um grafo bipartido completo $K_{p, q}$ é um grafo bipartido em $A$ e $B$, onde $|A|=p$ e $|B|=q$, tal que existe aresta $x y$ para todo par $x \in A, y \in B$.

Um caminho é uma sequência de vértices distintos $x_{1} x_{2} \ldots x_{\ell}$ de $G$ tal que existem as arestas $x_{i} x_{i+1} \in E$, para $1 \leq i \leq \ell-1$. Denote $P_{n}$ um caminho com $n$ vértices. O comprimento de um caminho é igual ao número de vértices do caminho menos uma unidade. Uma corda em um caminho é uma aresta de $G$ que interliga dois vértices não-consecutivos no caminho em questão. Um grafo é dito conexo se para cada par de vértices existe ao menos um caminho, e desconexo caso contrário. Dado um grafo conexo, um aresta é chamada de ponte se, quando removida do grafo, o torna desconexo. Um emparelhamento é um conjunto $M \subseteq E$ tal que todo vértice do grafo incide no máximo em uma aresta de $M$. Um componente conexo é um subgrafo maximal que induz um grafo conexo. Um caminho induzido é um caminho sem cordas. O diâmetro de um grafo é a maior distância entre qualquer par de vértices. Dado dois vértices $u, v \in V$, a distância entre $u e v$, denotada por $d_{G}(u, v)$, é o menor comprimento de um caminho induzido entre $u$ e $v$ no grafo. Um ciclo consiste de um caminho $x_{1} x_{2} \ldots x_{\ell}$ ao qual se adiciona a aresta $x_{1} x_{\ell}$. Denote por $C_{n}$ um ciclo com $n$ vértices, onde $C_{n}$ é um ciclo par (impar) se $n$ é um número par (ímpar). $\mathrm{O}$ ciclo $C_{3}$ é chamado de triângulo. Uma diagonal em ciclo é uma aresta de $G$ que interliga dois vértices não consecutivos do ciclo. Um ciclo induzido é um ciclo sem diagonais. O grafo $G$ é dito acíclico se não contém nenhum ciclo

\footnotetext{
${ }^{2}$ Podemos escrever $V(G)$ para reforçar que o conjunto de vértices é de $G$, assim como para as arestas podemos escrever $E(G)$.
} 
como subgrafo. Todo grafo conexo e acíclico é chamado de árvore; caso seja apenas acíclico, é chamado de floresta. Um grafo $K_{n}$ é dito completo se para cada par de vértices $u$ e $v$ de $K_{n}$ existe uma aresta. Uma clique é um subconjunto de vértices que induz um subgrafo completo; em outras palavras, cada conjunto de três vértices da clique induz num triângulo. Um vértice $v$ é chamado de simplicial se $N(v)$ é uma clique. Um grafo é dito split se podemos particionar seu conjunto de vértices em dois subconjuntos $A$ e $B$, onde $A$ é uma clique e $B$ é estável.

Uma propriedade importante é:

Propriedade 2.1. [21] Um grafo $G$ é bipartido se, e somente se, não contém ciclo impar como subgrafo induzido.

Como havíamos mencionado, a convexidade $P_{3}$ se baseia em caminhos curtos, isto é, caminhos cujo comprimento é 2 . Note que o vértice interno de qualquer caminho de comprimento 2 está na interseção entre as vizinhanças dos extremos do caminho. Assim, considere o operador $I_{3}$ aplicado em $a, b \in V$ sendo:

$$
I_{3}(a, b)=N[a] \cap N[b]
$$

Para um subconjunto de vértices $W$, considere $I_{3}[W]=\bigcup I_{3}(a, b)$ para todo $a, b \in W$, se $|W| \geq 2$, e $I_{3}[W]=W$ caso contrário. Dizemos que $W$ é $P_{3}$-convexo se, e somente se, $I_{3}[W]=W$. Assim, a convexidade $P_{3}$ é constituída por subconjuntos de $V$ que satisfazem a propriedade de serem $P_{3}$-convexos. O menor conjunto $P_{3^{-}}$ convexo contendo um dado $W$ é chamado de fecho $P_{3}$-convexo de $W$, e é denotado por $[W]_{p 3}$. O processo de encontrar o fecho $P_{3}$-convexo pode ser efetuado de forma iterativa. Para dado um $W \subseteq V$, considere:

i. $I_{3}^{1}[W]=I_{3}[W]$;

ii. $I_{3}^{k}[W]=I_{3}\left[I_{3}^{k-1}[W]\right]$.

O processo termina quando tivermos $I_{3}^{k}[W]=I_{3}^{k-1}[W]$ e, então, podemos concluir que $I_{3}^{k-1}[W]=[W]_{p 3}$. Pelo fato de o grafo ser finito, podemos concluir que todo o processo é finito.

Já para a convexidade $P_{3}^{*}$, considere o seguinte intervalo:

$$
I_{3}^{*}(a, b)=\left\{w \in V: d_{G}(a, w)+d_{G}(w, b)=2 \wedge d_{G}(a, b)=2\right\} \cup\{a, b\}
$$

Analogamente, para um subconjunto de vértices $W$, considere $I_{3}^{*}[W]=$ $\bigcup I_{3}^{*}(a, b)$ para todo $a, b \in W$, se $|W| \geq 2$, e $I_{3}^{*}[W]=W$ caso contrário. Dizemos que $W$ é $P_{3}^{*}$-convexo se, e somente se, $I_{3}^{*}[W]=W$. Assim, a convexidade $P_{3}^{*}$ é constituída por subconjuntos de $V$ que satisfazem a propriedade de serem $P_{3}^{*}$ convexos. O menor conjunto $P_{3}^{*}$-convexo contendo um dado $W$ é chamado de fecho $P_{3}^{*}$-convexo de $W$, e o escrevemos como $[W]_{p 3^{*}}$.

Podemos verificar que: 
Propriedade 2.2. Dado um grafo $G$, a convexidade $P_{3}$ definida sobre $G$ está contida na convexidade $P_{3}^{*}$ também definida sobre $G$.

Demonstração. Observe pelas definições dos intervalos das Eq (11) e Eq (2) que todo conjunto $P_{3}$-convexo é também $P_{3}^{*}$-convexo. Logo, vale a propriedade.

Um subconjunto $W$ de $V$ será chamado $P_{3}$-biconvexo $\left(P_{3}^{*}\right.$-biconvexo $)$ se $W$ e $\bar{W}$ são ambos $P_{3}$-convexos $\left(P_{3}^{*}\right.$-convexos). Note que é interessante que o subconjunto de vértices $W$ seja não-trivial, i.e., o conjunto $W$ tem que ser um subconjunto próprio e não-vazio de $V$. Portanto, como nosso interesse é o estudo apenas em partições não-triviais, omitiremos esse termo; porém, deixaremos claro que apenas importam subconjuntos próprios e não-vazios na partição. Além disso, podemos generalizar o problema com a utilização do termo $p$-partição $P_{3}$-convexas (ou $P_{3}^{*}$-convexas), onde o nosso interesse é particionar $V$ em $p$ subconjuntos disjuntos que sejam $P_{3}$-convexos (ou $P_{3}$-convexos). Note que biconvexo é uma particularização para $p=2$.

\section{ANÁLISE TEÓRICA E COMPUTACIONAL}

\subsection{Estudo Teórico de Partição e Separação Convexa}

Agora faremos análises teóricas de ambas as convexidades. Para entendermos a relação entre as duas convexidades, destacamos o primeiro resultado:

Teorema 3.1. Se $G$ possui um conjunto $W \subseteq V$ de modo que $W$ seja um conjunto $P_{3}$-biconvexo, então $W$ também é um conjunto $P_{3}^{*}$-biconvexo.

Demonstração. Pela Propriedade 2.2, temos que todo conjunto $P_{3}$-convexo é $P_{3}^{*}$ convexo. Assim, para dado $W P_{3}$-biconvexo, temos $W$ e $\bar{W}$ ambos $P_{3}$-convexos e, consequentemente, $W$ e $\bar{W}$ ambos $P_{3}^{*}$-convexos. Logo, $W$ é também $P_{3}^{*}$-biconvexo.

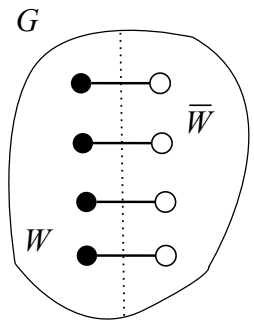

(i)

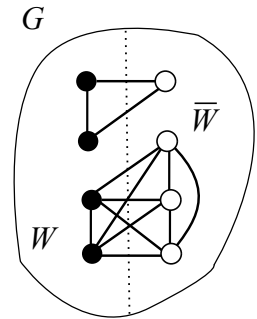

(ii)

Figura 1: Em $(i)$ o grafo $G$ contém $W P_{3}$-biconvexo, e em $(i i) W$ é $P_{3}^{*}$-biconvexo.

A recíproca do teorema anterior é falsa. Uma justificativa para tal é que um conjunto $W$ dito $P_{3}$-biconvexo não admite um vértice de $W$ que seja adjacente a dois em $\bar{W}$, assim como nenhum vértice em $\bar{W}$ possui dois vizinhos (adjacentes ou não) em $W$. Porém, um conjunto $P_{3}^{*}$-biconvexo admite a existência deste vértice se os seus vizinhos na outra parte forem adjacentes. Vejamos exemplos de partições válidas na Figura 1, onde em $(i)$ temos a exemplificação do comportamento das arestas para um conjunto $P_{3}$-biconvexo, e em $(i i)$ para um conjunto $P_{3}^{*}$-biconvexo.

Continuando no Teorema 3.1. podemos verificar aue: 
Corolário 3.1. Dado um grafo $G$, toda p-partição $P_{3}$-convexa para $G$ é também uma p-partição $P_{3}^{*}$-convexa para $G$.

Um fato interessante que podemos observar da Figura 1 é que as arestas da partição, i.e., as arestas que conectam $W$ a $\bar{W}$, são arestas de cliques. Para conjuntos $P_{3}$-biconvexos são exatamente arestas de cliques de tamanho 2 ; já para $P_{3}^{*}$-biconvexos são arestas de cliques de qualquer tamanho. Então, isso sugere que:

Proposição 3.1. Se $G$ não contém triângulos, as convexidades $P_{3}$ e $P_{3}^{*}$ são equivalentes para $G$.

Demonstração. Suponha que não sejam equivalentes as duas convexidades. Como foi verificado na Propriedade 2.2 temos que $W P_{3}$-convexo também é $P_{3}^{*}$-convexo. Então existe um conjunto $W$ que é $P_{3}^{*}$-convexo, mas que não é $P_{3}$-convexo. Então, isso significa que $I_{p 3}[W] \neq W$. Assim, temos que existe algum vértice $v$ em $I_{p 3}[W] \backslash$ $W$ de modo que $v$ é adjacente a pelo menos dois vértices em $W$. Mas $v$ não está em $I_{p 3^{*}}[W]$, pois $I_{p 3^{*}}[W]=W$. Assim, temos que $v$ tem arestas a dois vértices de $W$ adjacentes. Contradição, pois $G$ não contém triângulos.

Assim, podemos concluir que:

Corolário 3.2. Se $G$ não contém triângulos, então toda p-partição $P_{3}$-convexa é também uma p-partição $P_{3}^{*}$-convexa.

Observe que uma condição necessária para $G$ conter um conjunto $P_{3}$-biconvexo (ou $P_{3}^{*}$-biconvexo) é que $|V| \geq 2$. Se um grafo $G$ é desconexo, então podemos escolher $X=V\left(G^{\prime}\right)$ como um conjunto $P_{3}$-biconvexo (ou $P_{3}^{*}$-biconvexo), para $G^{\prime} \subseteq G$ conexo. Assim, considere mais duas importantes propriedades:

Propriedade 3.1. Seja $G$ um grafo conexo. Se $G$ possui ponte, então $G$ possui um conjunto $P_{3}$-biconvexo $W$ contendo um componente conexo com a remoção da ponte.

Propriedade 3.2. Seja $G$ um grafo conexo. Se existe um conjunto $P_{3}$-biconvexo $W \subseteq V$, então toda aresta de uma clique maximal de tamanho maior que 2 é induzida ou por $W$ ou por $\bar{W}$.

Ambas as propriedades podem ser verificadas pela Figura 1. Note que para a Propriedade 3.1 seria o caso de termos apenas uma aresta para a Figura 1 . $(i)$. A Propriedade 3.2 também pode ser verificada pelo mesmo item $(i)$ da figura; porém tal resultado não é verdade se compararmos com a Figura 1 - $(i i)$.

Agora, é fácil verificar para grafos como $P_{2}$ e $P_{3}$ que, por terem pontes, possuem um conjunto $P_{3}$-biconvexo. Além disso, $C_{3}=K_{3}$ não é $P_{3}$-biconvexo, pela Propriedade 3.2, Logo:

Corolário 3.3. $C_{3}$ é o menor grafo que não admite um conjunto $P_{3}$-biconvexo.

Note que qualquer vértice simplicial pode particionar qualquer grafo na convexidade $P_{0}^{*}$. Assim. também podemos verificar aue: 
Propriedade 3.3. Seja $G$ um grafo conexo. Se $G$ possui um vértice simplicial, então existe um conjunto $P_{3}^{*}$-biconvexo em $G$.

Assim, verificamos que:

Corolário 3.4. $K_{2,3}$ é o menor grafo que não admite um conjunto $P_{3}^{*}$-biconvexo. Demonstração. Pela Propriedade 3.3. temos que não existem vértices simpliciais no grafo que buscamos sem partição $P_{3}^{*}$-biconvexa. Então $C_{3}$ deve ser descartado. Aliás, qualquer grafo com 4 vértices também deve ser descartado, pois sempre teremos um conjunto $P_{3}^{*}$-biconvexo neste caso, visto que tal grafo não contém pontes e nem vértices simpliciais pela Propriedade 3.1 e pela Propriedade 3.3 (note que $C_{4}$ admite um conjunto $P_{3}^{*}$-biconvexo, colocando dois vértices adjacentes em $W$ ). Então, o menor grafo que não admite um conjunto $P_{3}^{*}$-biconvexo tem 5 vértices. Podemos verificar que $K_{2,3}$ não admite tal conjunto. No entanto, a remoção de qualquer aresta leva a um grafo com partição biconvexa, pois haverá uma ponte após a remoção. Logo, $K_{2,3}$ é o menor grafo que não admite um conjunto $P_{3}^{*}$-biconvexo.

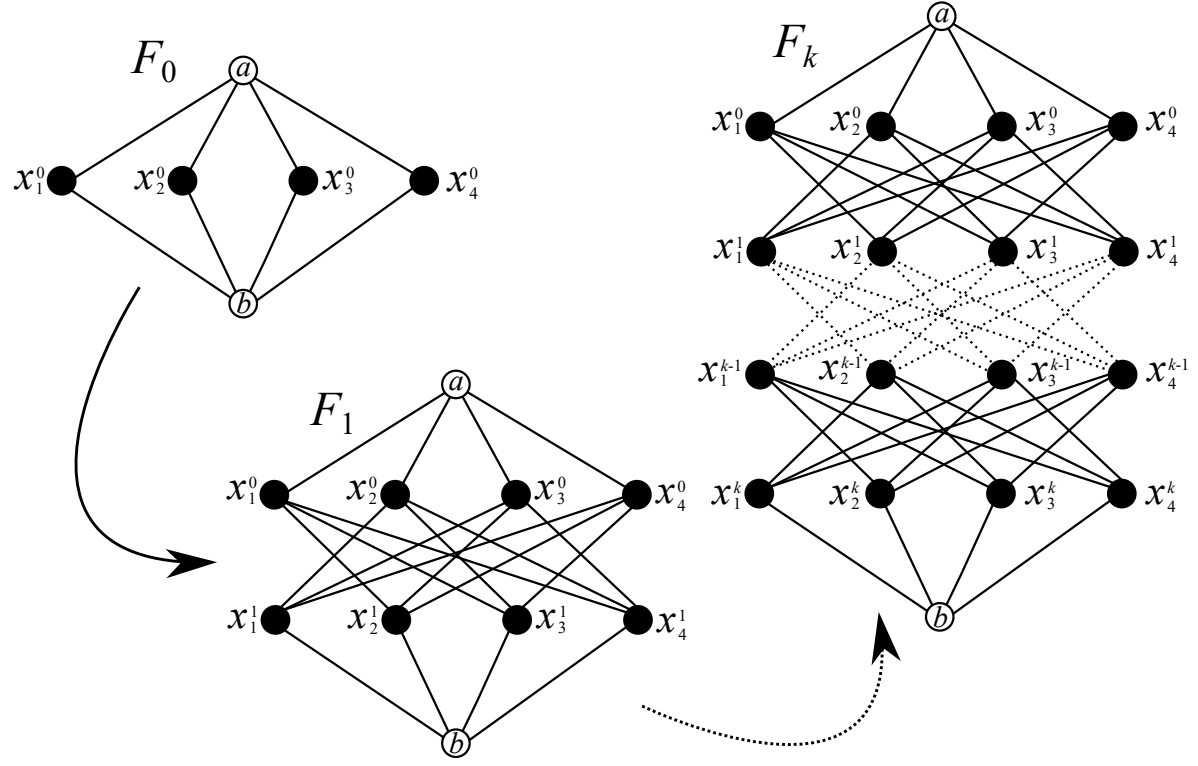

Figura 2: Família infinita de grafos que não admitem conjuntos $P_{3}$-biconvexos nem $P_{3}^{*}$-biconvexos.

Na verdade, existe uma infinidade de grafos com a característica de não admitirem uma partição biconvexa. Para isso, primeiro considere $F_{0}$ como na Figura 2. Note que este grafo é $K_{2,4}$, que contém $K_{2,3}$. Para construir $F_{1}$, removemos todas as arestas incidentes a $b$ e criamos 4 vértices $x_{1}^{1}, x_{2}^{1}, x_{3}^{1}, x_{4}^{1}$ fazendo-os adjacentes a $b$; em seguida criamos o conjunto de arestas $\left\{x_{i}^{0} x_{j}^{1}: 1 \leq i, j \leq 4 \wedge i \neq j\right\}$. Assim, temos $F_{1}$ conforme mostra a Figura 2, Repetindo o processo $k$ vezes, removendo todas as arestas incidentes a $b$ e criando 4 vértices $x_{1}^{k}, x_{2}^{k}, x_{3}^{k}, x_{4}^{k}$ fazendo-os adjacentes a $b$, e inserindo as arestas $\left\{x_{i}^{k-1} x_{j}^{k}: 1 \leq i, j \leq 4 \wedge i \neq j\right\}$, criamos $F_{k}$. Note que $F_{i}$ não está contido em $F_{k}$, para todo $i<k$ inteiros não-negativos.

Considere o lema a seguir: 
Lema 3.1. O grafo $F_{k}$ não admite um conjunto $P_{3}$-biconvexo e nem $P_{3}^{*}$-biconvexo, para todo $k$ inteiro não-negativo.

Demonstração. Mostraremos por indução que $F_{k}$ não é $P_{3}$-biconvexo, para todo $k$ inteiro não-negativo. A base de indução está satisfeita pelo Corolário 3.4, visto que $F_{0}$ é isomorfo a $K_{2,4}$, que é composto por $K_{2,3}$ mais um vértice adjacente aos vértices da menor parte. Considere $F_{k}$, para $k>0$ inteiro não-negativo. Fazendo $V\left(F_{k}\right) \backslash\left(\left\{b, x_{\alpha}^{k}, x_{\beta}^{k}, x_{\gamma}^{k}\right\}\right)$ e $x_{\delta}^{k}$ adjacente a $x_{\delta}^{k-1}$, temos um grafo isomorfo a $F_{k-1}$, para $\alpha, \beta, \gamma \in\{1,2,3,4\}$ e $\delta \in\{1,2,3,4\} \backslash\{\alpha, \beta, \gamma\}$. Pela hipótese de indução, tal grafo não admite um conjunto $P_{3}$-biconvexo. Com isso temos que qualquer tentativa de encontrar um conjunto $W P_{3}$-biconvexo com ou sem $x_{\delta}^{k}$ é frustrada, pois ou $[W]_{p 3}=V$ ou $[\bar{W}]_{p 3}=V$. No entanto, sem a aresta $x_{\delta}^{k} x_{\delta}^{k-1}$, poderíamos verificar ou $[W]_{p 3} \neq V$ ou $[\bar{W}]_{p 3} \neq V$, para algum $W$. Para cada $W \subseteq V\left(F_{k}\right) \backslash\left(\left\{b, x_{\alpha}^{k}, x_{\beta}^{k}, x_{\gamma}^{k}\right\}\right)$, vejamos os seguintes casos para $F_{k}$ :

- Se $x_{\delta}^{k} \in[W]_{p 3}$, temos pelo menos $x_{\alpha^{\prime}}^{k-1}, x_{\beta^{\prime}}^{k-1} \in N\left(x_{\delta}^{k}\right)$ que estão em $[W]_{p 3}$. No entanto, também temos $x_{\gamma^{\prime}}^{k}$ na mesma situação de $x_{\delta}^{k}$ e, com isso, podemos concluir que $x_{\gamma^{\prime}}^{k} \in[W]_{p 3}$. Então, temos $b \in[W]_{p 3}$, pois $b \in I_{3}\left(x_{\gamma^{\prime}}^{k}, x_{\delta^{\prime}}^{k}\right)$. Logo $x_{\alpha^{\prime}}^{k}, x_{\beta^{\prime}}^{k} \in[W]_{p 3}$, pois $x_{\alpha^{\prime}}^{k} \in I_{3}\left(x_{b^{\prime}}^{k-1}, b\right)$ e $x_{\beta^{\prime}}^{k} \in I_{3}\left(x_{\alpha^{\prime}}^{k-1}, b\right)$, e por esta razão temos $x_{\alpha}^{k-1}, x_{\beta}^{k-1}, x_{\gamma}^{k-1}, x_{\delta}^{k-1} \in W$. Portanto, $[W]_{p 3}=V$.

- Se $x_{\delta}^{k} \notin[W]_{p 3}$, temos pelo menos $x_{\alpha^{\prime}}^{k-1}, x_{\beta^{\prime}}^{k-1} \in N\left(x_{\delta}^{k}\right)$ que não estão em $[W]_{p 3}$. De forma análoga ao item anterior, chegaremos à conclusão de que $b$ não está em $[W]_{p 3}$, assim como os restantes. Logo $[\bar{W}]_{p 3}=V$.

Agora, se $W \subseteq\left\{b, x_{\alpha}^{k}, x_{\beta}^{k}, x_{\gamma}^{k}\right\}$, temos um grafo $K_{2,3}$ para cada vértice $x_{1}^{k-1}, x_{2}^{k-1}, x_{3}^{k-1}, x_{4}^{k-1}$. Assim, esses vértices ou inserem ou removem vértices de $V\left(F_{k-1}\right)$. Logo, para $F_{k}$, ou $[W]_{p 3}=V$ ou $[\bar{W}]_{p 3}=V$, para todo $W \subseteq V\left(F_{k}\right)$.

Para finalizar, note que $F_{k}$ não contém triângulos, para todo $k$ inteiro nãonegativo, e temos que todo conjunto $P_{3}^{*}$-convexo é $P_{3}$-convexo pela Proposição 3.1 Assim, concluímos a prova.

Podemos verificar que:

Teorema 3.2. Existem infinitos grafos que não são $P_{3}$-biconvexos nem $P_{3}^{*}$-biconvexos.

Este resultado era esperado, visto que verificar existência de partição para a convexidade $P_{3}$ é um problema NP-completo, como discutiremos na próxima sessão.

Agora, veremos alguns resultados sobre separação. Como este é um tema até o momento pouco estudado em grafos, trazemos alguns aspectos preliminares em algumas classes pequenas.

Lema 3.2. Para todo caminho induzido $P_{n}$ com $n \geq 2$, temos que a convexidade $P_{3}$ é uma $s_{3}$-convexidade. 
Demonstração. Seja $P_{n}$ induzido com $n \geq 2$. Considere $W$ um conjunto $P_{3}$-convexo e $v \in V \backslash W$. Se o grau de $v$ for 1 , podemos notar que $S=V \backslash\{v\}$ é $P_{3}$-biconvexo. Caso contrário, note que $v$ não possui dois vizinhos em $X$. Assim, podemos impedir que um vizinho de $v^{\prime}$ de $v$ entre em $S$. Portanto, $S=V \backslash\left\{v, v^{\prime}\right\}$ é $P_{3}$-biconvexo.

Vale lembrar que um conjunto convexo não precisa ser conexo necessariamente. O axioma $S_{4}$ não é válido para qualquer caminho, pois se tomarmos $P_{5}: v_{1} v_{2} v_{3} v_{4} v_{5}$ e fizermos $W=\left\{v_{1}, v_{4}\right\}$ e $W^{\prime}=\left\{v_{2}, v_{5}\right\}$ conjuntos $P_{3}$-convexos, não há conjunto $P_{3}$-biconvexo $S$ de modo que $W \subseteq S$ e $W^{\prime} \subseteq V \backslash S$. Isso se deve ao fato de $v_{3}$ afetar os conjuntos $W$ e $W^{\prime}$ quando tentamos colocá-lo em $S$ ou fora dele.

Teorema 3.3. Para toda árvore $T_{n}$ com $n \geq 2$, temos que a convexidade $P_{3}$ é uma $s_{3}$-convexidade.

Demonstração. Se $T_{n}$ for um caminho, temos a prova concluída pelo Lema 3.2. Caso contrário, dados $W \subseteq V$ convexo e $v \in V \backslash W$, temos que o vértice $v$ não tem dois vizinhos em $W$. Portanto, façamos $W^{\prime}=N[v] \backslash W$. Assim, podemos concluir que $S=V \backslash W^{\prime}$ é $P_{3}$-biconvexo.

Do teorema anterior e da Proposição 3.1 podemos concluir que:

Corolário 3.5. Para toda árvore $T_{n}$ com $n \geq 2$, temos que a convexidade $P_{3}^{*}$ é uma $s_{3}$-convexidade.

Teorema 3.4. Para todo ciclo induzido $C_{n}$ com $n \geq 4$, temos que a convexidade $P_{3}$ é uma $s_{3}$-convexidade.

Demonstração. A prova é análoga à do Lema 3.2. onde o grau de $v$ é igual a dois.

Do teorema anterior e da Proposição 3.1. podemos concluir que:

Corolário 3.6. Para todo ciclo induzido $C_{n}$ com $n \geq 4$, temos que a convexidade $P_{3}^{*}$ é uma $s_{3}$-convexidade.

Já para o grafo $C_{n}, \operatorname{com} n \geq 4$, não podemos dizer que as convexidades $P_{3}$ ou $P_{3}^{*}$ são $s_{4}$-convexidades. Para isso, tomamos $C_{7}: v_{1} v_{2} v_{3} v_{4} v_{5} v_{6} v_{7}$, com $W=\left\{v_{1}, v_{4}\right\}$ e $W^{\prime}=\left\{v_{2}, v_{5}\right\}$. Note que $W$ e $W^{\prime}$ são ambos convexos. Todavia, não há como encontrar $S P_{3}$-biconvexo (ou $P_{3}^{*}$-biconvexo) com ou sem o vértice $v_{3}$. Portanto, em grafos restritos a ciclos de tamanho maior que 3 , as convexidades $P_{3}$ e $P_{3}^{*}$ não são $s_{4}$-convexidades.

Teorema 3.5. Para toda clique $K_{n}$, com $n \geq 3$, temos que a convexidade $P_{3}$ é uma $s_{1}$-convexidade.

Demonstração. A prova é bem simples, pois temos da Propriedade 3.2 que todos os vértices de uma clique maximal de tamanho maior que 2 têm que pertencer ao mesmo conjunto $P_{3}$-biconvexo. Logo, não há como particionar $K_{n}$ e, portanto, temos que os axiomas $S_{2}, S_{3}$ e $S_{4}$ não são válidos por dependerem de um conjunto $P_{3}$-biconvexo. Logo, segue o teorema.

\footnotetext{
${ }^{3}$ Nos primórdios de estudo da convexidade em grafos, exigia-se a conexidade dos elementos da convexidade. mas isto foi perdendo importância à medida que surgiam novas convexidades.
} 
Contudo, temos o seguinte resultado:

Teorema 3.6. Para toda clique $K_{n}$, com $n \geq 2$, temos que a convexidade $P_{3}^{*}$ é uma $s_{4}$-convexidade.

Demonstração. Pela Propriedade 3.3, temos que os vértices simpliciais particionam o grafo em conjuntos $P_{3}^{*}$-biconvexos. Dados $W$ e $W^{\prime}$ convexos e disjuntos, façamos $S=W$. Note que todos os vértices de $S$ são simpliciais em $S$, assim como os vértices de $V\left(K_{n}\right) \backslash S$. Logo, podemos verificar que $S$ é $P_{3}^{*}$-biconvexo.

Sobre separação, temos a seguinte propriedade:

Propriedade 3.4. [2] Para os axiomas $s_{1}, s_{2}, s_{3}$ e $s_{4}$ temos:

i. $s_{2} \Rightarrow s_{1}$;

ii. Assumindo $s_{1}$ verdadeiro, então $s_{4} \Rightarrow s_{3} \Rightarrow s_{2}$.

Lembramos que o conjunto unitário também é convexo em ambas as convexidades estudadas. Logo, pela a propriedade acima e o Teorema $\mathbf{3 . 6}$, temos:

Corolário 3.7. A convexidade $P_{3}^{*}$ tem uma separação em grafos completos.

Apesar do resultado acima, os grafos completos de tamanho maior que 2 constituem um família infinita de conjuntos que não admitem conjuntos $P_{3^{-}}$ biconvexos. Assim, podemos concluir que a quantidade de grafos que não admitem um conjunto $P_{3}$-biconvexo é bem maior do que os que não admitem um conjunto $P_{3}^{*}$-biconvexo.

\subsection{Análise Computacional}

A análise de complexidade é algo inerente ao nosso objeto de estudo. Existem problemas combinatórios que se relacionam com os problemas deste trabalho, e, por serem relevantes, foram colocados nesta seção para reforçar os resultados de complexidade, alguns já existentes na literatura.

Assim, formalizamos:

Problema da $p$-PARTiÇÃo $P_{3}$-CONVEXa $\left(\mathrm{P}_{3} \mathrm{PC}\right)$

Instância: Um grafo conexo $G$ e um inteiro positivo $p$.

Questão: $G$ possui uma p-partição em conjuntos $P_{3}$-convexos?

E, analogamente:

Problema $p$-PARTiÇÃo $P_{3}^{*}$-CONVEXA $\left(\mathrm{P}_{3}^{*} \mathrm{PC}\right)$

Instância: Um grafo conexo $G$ e um inteiro positivo $p$.

Questão: $G$ possui uma p-partição em conjuntos $P_{3}^{*}$-convexos? 
Para ambos os casos, os problemas em partição biconvexa são denotados como $P_{3} 2 C$ e $P_{3}^{*} 2 C$, respectivamente.

Como mencionamos, o problema $\mathrm{P}_{3} \mathrm{PC}$ é NP-completo, mesmo para grafos split [20]. Contudo, veremos que existem outras classes de grafos onde o problema permanece NP-completo. Para $E^{\prime} \subseteq E$, denote por $G-E^{\prime}$ o grafo resultante da remoção das arestas em $E^{\prime}$ de $G$. Assim, considere o seguinte problema:

Problema de Emparelhamento de Corte ("Matching-Cut" - MC)

Instância: Um grafo conexo $G$.

Questão: Existe um emparelhamento $E^{\prime} \subseteq E$ tal que $G-E^{\prime}$ é desconexo?

O problema MC é NP-completo, mesmo para grafos planares com grau máximo 4 [22, 23, 24], ou grafos com diâmetro máximo $d$ fixo $(d \geq 4)$ [25].

Podemos verificar que, em essência, ambos os problemas $\mathrm{MC}$ e $\mathrm{P}_{3} 2 \mathrm{C}$ são equivalentes. Assim, podemos verificar:

Teorema 3.7. O problema $\mathrm{P}_{3} 2 \mathrm{C}$ é NP-completo, mesmo restrito a grafos planares com grau máximo 4 ou com diâmetro máximo $d$ fixo, com $d \geq 4$.

Note que se um grafo $G$ possui uma $p$-partição em conjuntos $P_{3}$-convexos, então $G$ possui um conjunto $P_{3}$-biconvexo. Logo:

Corolário 3.8. O problema $\mathrm{P}_{3} \mathrm{PC}$ com $p$ fixo é NP-completo, mesmo restrito a grafos planares com grau máximo 4 ou grafos com diâmetro máximo d fixo, com $d \geq 4$.

Enunciamos agora o seguinte teorema:

Teorema 3.8. O problema $\mathrm{P}_{3}^{*} 2 \mathrm{C}$ é NP-completo.

Demonstração. Primeiro, é fácil verificar um certificado em tempo polinomial. Para isso, basta analisar se $W \neq \emptyset$ e $W \neq V$ e, em seguida, efetuar o intervalo da Eq 2 para cada par de vértices $a, b$ em $W$, a fim de verificar se $I_{3}^{*}(a, b) \subseteq W$. Como isso pode ser feito em tempo polinomial, o problema está em NP.

Considere $\langle G=(V, E)\rangle$ um entrada para o problema $\mathrm{P}_{3} 2 \mathrm{C}$, e construamos a partir dela uma entrada $\left\langle G^{*}=\left(V^{*}, E^{*}\right)\right\rangle$ para o problema $\mathrm{P}_{3}^{*} 2 \mathrm{C}$. A função transformação $T(G)$ consiste em eliminar os triângulos de $G$, pois pela Proposição 3.1 garantimos que ambas as convexidades são equivalentes neste caso. Para isso, para cada $\{a, b, c\} \subseteq V$ induzindo $C_{3}$ em $G$, criaremos o conjunto de vértices $\left\{a, b, c, v_{a b}, v_{a c}, v_{b c}, u_{a b c}, u_{a b c}^{\prime}\right\}$ em $V^{*}$, o conjunto das arestas $\left\{a v_{a b}, a v_{a c}, b v_{a b}, b v_{b c}, c v_{a c}\right.$, $\left.a v_{b c}, v_{a b} u_{a b c}, v_{a b} u_{a b c}^{\prime}, v_{b c} u_{a b c}, v_{b c} u_{a b c}^{\prime}, v_{a c} u_{a b c}, v_{a c} u_{a b c}^{\prime}\right\}$ em $E^{*}$, e removemos as arestas $a b, a c$ e $b c$. Essa transformação de $G$ para $G^{*}$ pode ser vista na Figura 3 .

Para os vértices restantes que não pertencem a nenhum triângulo, replicamolos em $G^{*}$ mantendo as mesmas adjacências do grafo $G$. Note que a transformação $T(G)$ é feita em tempo polinomial. 

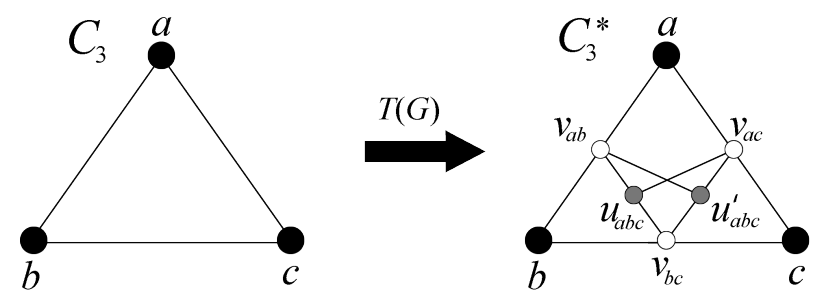

Figura 3: Método da transformação $T(G)=G^{*}$, que converte os triângulos de $G$, à esquerda, nas estruturas em $G^{*}$, à direita.

Agora seguiremos com a conclusão da prova de equivalência entre os problemas.

$(\Rightarrow)$ Seja $\langle G=(V, E)\rangle$ uma instância SIM do problema $\mathrm{P}_{3} 2 \mathrm{C}$. Seja $W$ um conjunto $P_{3}$-biconvexo de $G$. Então, pelo Teorema 3.1, temos que $W$ também é um conjunto $P_{3}^{*}$-biconvexo. Assim sendo, se existe um subgrafo $C_{3}$ induzido por $W$, então fazemos $W^{*}$ receber os vértices do subgrafo associado $C_{3}^{*}$ pela função transformação $T(G)$. Já os vértices restantes de $W$ também são associados a seus correspondentes em $W^{*}$ por $T(G)$. Um fato importante: pela Propriedade 3.2 , temos que não existe nenhum subgrafo $C_{3}$ com algum vértice em $W$ e outro em $\bar{W}$. Logo, os vértices de um subgrafo $C_{3}^{*}$ não podem ser compartilhados entre $W^{*}$ e $\bar{W}^{*}$. Assim, podemos concluir que $W^{*}$ é um conjunto $P_{3}^{*}$-biconvexo. Portanto, $\left\langle G^{*}=\left(V^{*}, E^{*}\right)\right\rangle$ é uma instância SIM do problema $\mathrm{P}_{3}^{*} 2 \mathrm{C}$.

$(\Leftarrow)$ Seja $\left\langle G^{*}=\left(V^{*}, E^{*}\right)\right\rangle$ uma instância SIM do problema $\mathrm{P}_{3}^{*} 2 \mathrm{C}$. Seja $W^{*}$ um conjunto $P_{3}^{*}$-biconvexo de $G^{*}$. Agora, considere a seguinte afirmação:

Afirmação 1: Para cada $C_{3}^{*}$ criado pela função transformação $T(G)$, temos que não existe nenhum conjunto $P_{3}^{*}$-biconvexo em $G$ de modo a não conter todos os vértices de $C_{3}^{*}$.

Isto é, se algum vértice em $C_{3}^{*}$ pertencer a um conjunto $W P_{3}^{*}$-biconvexo, então todos vértices de $C_{3}^{*}$ pertencem a $W$. Isso se deve ao fato de que todos os vértices de $C_{3}^{*}$ pertencem a algum subgrafo $K_{2,3}$, que não possui conjunto $P_{3}^{*}$ biconvexo pelo Corolário 3.4. Note que pela Proposição 3.1. temos que $W^{*}$ também é um conjunto $P_{3}$-biconvexo, pois $G^{*}$ não contém triângulo por construção. Além disso, temos que os vértices de cada $C_{3}^{*}$ de $G^{*}$ pertencem ou a $W^{*}$ ou a $\bar{W}^{*}$ pela Afirmação 1. Então, se fizermos $W$ receber todos os vértices originais de $G$ em $W^{*}$, teremos que os triângulos de $G$ nunca estarão compartilhados entre $W$ e $\bar{W}$, e com isso estaremos apenas ligando pares distintos entre $W$ e $\bar{W}$. Portanto, temos uma instância SIM para $\langle G=(V, E)\rangle$.

Note que se $G$ tiver grau máximo 4, a transformação $T(G)$ não muda o grau dos vértices em $G^{*}$, e os vértices novos possuem grau 3 . Portanto, o problema $\mathrm{P}_{3}^{*} 2 \mathrm{C}$ permanece NP-completo mesmo quando o grafo tem grau máximo 4. Assim:

Corolário 3.9. O problema $\mathrm{P}_{3}^{*} \mathrm{PC}$ com p fixo é NP-completo, mesmo para grafos com grau máximo 4 .

Corolário 3.10. O problema $\mathrm{P}_{\curvearrowright}^{*} 2 \mathrm{C}$ é $N P$-completo para arafos bipartidos. 
Demonstração. Considere a mesma construção do Teorema 3.8 para arestas em triângulos, e para as arestas restantes $x y$ criaremos os vértices $z_{x y}$ e $z_{x y}^{\prime}$ com as arestas $\left\{x z_{x y}, x z_{x y}^{\prime}, y z_{x y}, y z_{x y}^{\prime}\right\}$. Note que removemos todos os ciclos ímpares. Assim, se existir um conjunto $P_{3}$-biconvexo $W$ em $G$, façamos $r, z_{r s}$ em $W^{*}$ e $s, z_{r s}^{\prime}$ em $\bar{W}^{*}$, onde $r \in W$ e $s \in \bar{W}$. Já para os outros vértices façamos $r, s, z_{r s}, z_{r s}^{\prime}$ em $W^{*}$ se $r, s \in W$; ou $r, s, z_{r s}, z_{r s}^{\prime}$ em $\bar{W}^{*}$ caso contrário. E, por fim, temos $W^{*}$ um conjunto $P_{3}^{*}$-biconvexo. Em contrapartida, se $G^{*}$ contém um conjunto $P_{3}^{*}$-biconvexo $W^{*}$, temos que um vértice em $W^{*}$ não possui mais de um vizinho em $\bar{W}^{*}$. Com isso, podemos encontrar um conjunto $P_{3}$-convexo $W$ fazendo $W=W^{*} \cap V(G)$ e $\bar{W}=\bar{W}^{*} \cap V(G)$, e assim podemos notar que, para qualquer aresta $r s$ cujos extremos satisfazem $r \in W$ e $s \in \bar{W}$, ocorre ou $z_{r s} \in W^{*}$ e $z_{r s}^{\prime} \in \bar{W}^{*}$, ou $z_{r s}^{\prime} \in W^{*}$ e $z_{r s} \in \bar{W}^{*}$. Logo, $G$ possui um conjunto $P_{3}$-biconvexo, se e somente se, $G^{*}$ possui um conjunto $P_{3}^{*}$-biconvexo Portanto, o problema permanece NP-completo.

Corolário 3.11. O problema $\mathrm{P}_{3}^{*} \mathrm{PC}$ com p fixo é NP-completo para grafos bipartidos.

Novamente, esse resultado para o problema $\mathrm{P}_{3}^{*} \mathrm{PC}$ se relaciona ao problema $\mathrm{MC}$, pois também o problema MC foi demonstrado NP-completo para grafos planares bipartidos [24], ou grafos bipartidos com grau máximo 4 [26].

\section{CONCLUSÃO}

Embora os problemas que estudamos se enlaçam pelo fato de que os conjuntos da convexidade $P_{3}$ estão contidos na convexidade $P_{3}^{*}$ definida sobre o mesmo grafo, e que elas se equivalem quando o grafo não possui triângulos, verificamos diversos outros aspectos interessantes registrados neste trabalho. Identificamos os menores subgrafos que não admitem um conjunto $P_{3}$-biconvexo e $P_{3}^{*}$-biconvexo, mostramos uma construção sistemática de uma lista infinita de grafos que não admitem conjuntos $P_{3}$-biconvexo nem $P_{3}^{*}$-biconvexos, e analisamos os casos de separação para caminhos, árvores, ciclos e cliques.

Ademais, trouxemos alguns resultados computacionais de NP-completude para partição convexa em ambas as convexidades, mesmo para grafos bipartidos.

E assim, notamos que os resultados para as convexidades permanecem NPcompletos mesmo para grafos planares com grau máximo 4 (exceto $\mathrm{P}_{3}^{*} \mathrm{PC}$ ), planares bipartidos, bipartidos com grau máximo 4 e grafos com diâmetro máximo $d$ fixo $(d \geq 4)$.

Em trabalhos futuros, pretendemos analisar os aspectos teórico-computacional do problema da $p$-partição convexas na convexidade $P_{3}$ e na convexidade $P_{3}^{*}$ sobre classes particulares de grafos, tais como cordais, cografos, grafos $P_{4}$-tidy, grafos de distância-hereditária etc. Além disso, pretendemos nos aprofundar mais sobre o tema de separação convexa em classes de grafos maiores do que foram apresentadas neste trabalho.

Reconhecimento. Os autores são gratos a FAPERJ (Ed. Sediadas/2018), CNPq e CAPES pelo suporte financeiro. 


\section{REFERÊNCIAS BIBLIOGRÁFICAS}

[1] BERGER, M. Convexity. Amer. Math. Monthly, v. 97, n. 8, p. 650 - 678, 1990. 2

[2] VAN DE VEL, M. L. J. Theory of Convex Structures. 1st edition. ed. Amsterdam: North Holland, 1993. 2, 11

[3] MOSCARINI, M.; MALVESTUTO, F. M. Two classes of graphs in which some problems related to convexity are efficiently solvable. Discrete Mathematics, Algorithms and Applications, v. 10, n. 03, p. 1850042, 2018. 2

[4] MATHEW, J. K.; MATHEW, S. Monophonic convexity in weighted graphs. Discrete Mathematics, Algorithms and Applications, v. 10, n. 01, p. 1850010, 2018. 2

[5] MARCILON, T.; SAMPAIO, R. The maximum infection time of the p3 convexity in graphs with bounded maximum degree. Discrete Applied Mathematics, v. 251, p. $245-257,2018.2$

[6] MORDESON, J. N.; MATHEW, S. Distances and convexity in fuzzy graphs. In: . Advanced Topics in Fuzzy Graph Theory. Cham: Springer International Publishing, 2019. p. 93-126. 2

[7] COELHO, E. M. et al. On the p3-hull number of some products of graphs. Discrete Applied Mathematics, v. 253, p. 2 - 13, 2019. 14th Cologne-Twente Workshop on Graphs and Combinatorial Optimization (CTW 2016). 2

[8] HARARY, F.; NIEMINEN, J. Convexity in graphs. J. Differential Geom, v. 16, n. 2 , p. $185-190,1981.3$

[9] FARBER, M.; JAMISON, R. E. Convexity in graphs and hypergraphs. SIAM Journal on Algebraic Discrete Methods, v. 7, n. 3, p. 433-444, 1986. 3

[10] DUCHET, P. Convex sets in graphs, ii. minimal path convexity. Journal of Combinatorial Theory, Series B, v. 44, n. 3, p. 307 - 316, 1988. 3

[11] CHANGAT, M.; MATHEW, J. On triangle path convexity in graphs. Discrete Mathematics, v. 206, p. $91-95,1999.3$

[12] CáCERES, J.; MáRQUEZ, A.; PUERTAS, M. L. Steiner distance and convexity in graphs. European Journal of Combinatorics, v. 29, n. 3, p. 726 - 736, 2008. 3

[13] PELAYO, I. M. Geodesic Convexity in Graphs. 1st edition. ed. New York: Springer, 2013. 3

[14] PENSO, L. D. et al. Complexity analysis of p3-convexity problems on boundeddegree and planar graphs. Theoretical Computer Science, v. 607, p. 83-95, 2015. 3 
[15] Centeno, C. C.; DOURADO, M. C.; SZWARCFiter, J. L. On the convexity of paths of length two in undirected graphs. Electronic Notes in Discrete Mathematics, v. 32, p. 11 - 18, 2009. DIMAP Workshop on Algorithmic Graph Theory. 3

[16] ARAúJO, R. T.; SAMPAIO, R. M.; SZWARCFITER, J. L. The convexity of induced paths of order three. Electronic Notes in Discrete Mathematics, v. 44, p. $109-114,2013.3$

[17] GIMBEL, J. Some remarks on the convexity number of a graph. Graphs and Combinatorics, v. 19, n. 3, p. 357-361, 2003. 3

[18] DOURADO, M. C.; PROTTI, F.; SZWARCFITER, J. L. Complexity results related to monophonic convexity. Discrete Applied Mathematics, v. 158, n. 12, p. 1268 - 1274, 2010. 3

[19] ARTIGAS, D. et al. Partitioning a graph into convex sets. Discrete Mathematics, v. 311, n. 17, p. 1968 - 1977, 2011. 3

[20] CENTEnO, C. C. et al. Convex partitions of graphs induced by paths of order three. Discrete Mathematics $\&$ Theoretical Computer Science, v. 12, n. 5, p. 175184, 2010. 3, 12

[21] BONDY, A.; MURTY, M. R. Graph theory. 1st edition. ed. London: Springer, 2008. 5

[22] CHVÁtAL, V. Recognizing decomposable graphs. Journal of Graph Theory, v. 8, n. 1, p. $51-53,1984.12$

[23] PATRIGNANI, M.; PIZZONIA, M. The complexity of the matching-cut problem. In: BRANDSTÄDT, A.; LE, V. B. (Ed.). Graph-Theoretic Concepts in Computer Science. Berlin, Heidelberg: Springer Berlin Heidelberg, 2001. p. 284295. 12

[24] BONSMA, P. The complexity of the matching-cut problem for various graph classes. Electronic Notes in Discrete Mathematics, v. 13, p. 18 - 21, 2003. 12, 14

[25] HOANG-OANH LE; BANG LE, V. On the Complexity of Matching Cut in Graphs of Fixed Diameter. In: HONG, S.-H. (Ed.). 27th International Symposium on Algorithms and Computation (ISAAC 2016). Dagstuhl, Germany: Schloss Dagstuhl-Leibniz-Zentrum fuer Informatik, 2016. (Leibniz International Proceedings in Informatics (LIPIcs), v. 64), p. 50:1-50:12. 12

[26] BANG LE, V.; RANDERATH, B. On stable cutsets in line graphs. Theoretical Computer Science, v. 301, n. 1, p. $463-475,2003.14$ 